\title{
Performance Research Of Wimax Networks With Frame Periods In Strict Priority
}

\author{
Khan Mubeen Ahmed, Bandhu Kailash Chandra
}

\begin{abstract}
WiMAX Networks is widely using today since the use of data and internet is increasing day by day. Availability of network all the time is a bigger challenge today. Performance of network depends on a number of factors and these parameters could play a wide role in the enhancement of coverage in the network. In this paper one important factor frame period is analyzed in WiMAX networks. Increasing the frame period in WiMAX network is analyzed in this paper and its performance is evaluated. A comparative study of Strict Priority is done in this paper with two different frame periods and it is observed that increasing frame period degrades the performance of WiMAX networks. This Analysis could be helpful for researches and Industrialist for real implementations.
\end{abstract}

Index Terms: 802.16, Light WiMAX Simulator (LWX), Bandwidth Allocation Algorithm (BWA), Strict Priority (SP), Frame Period (FP).

\section{INTRODUCTION}

Solution for rapid requirement of internet for data and voice services are Broadband Wireless Access (BWA). Alternative solution of cable

Network and DSL Digital Subscriber line is also BWA [1]. The IEEE working group has designed IEEE 802.16 standard based on BWA systems [2]. High data rate deployment, large area coverage and to use large frequency spectrums in the available networks, it can be used. It offers integrated voice, video and data services. It is suitable for rural areas and crowded geographical areas. Here one Base Station (BS) and many Subscriber Stations (SSs) are used for topology. Clients are connected to SS for data transfer or any SS can itself be a client. Synchronization of SSs with BS is necessary for data transmission. The BS communicated to all SS in the beginning of each

Frame via uplink Map (UL MAP) [3]. The contribution of LWX is in the area mainly focused on QoS, OFDMA, and multi hop relay. The mechanism provided by LWX for user to plug and play different algorithms. One of the important research areas is to allocate a suitable algorithm, and to change the existing parameters with the proposed parameter this field and analyze the result.

\section{BANDWIDTH ALLOCATION ALGORITHM}

Strict Priority scheduling may be preemptive or may be non preemptive. One Important feature of priority scheduling is that it suffers the problem of starvation. A process losses control of the base station through one of the following task completion, a higher priority task becoming ready or in a wait condition [7]. When Higher Priority processes are ready to process, than lower priority processes are neglected. In non priority preemptive scheduling higher priority process took a long time on the base station could creates a starvation environment.

\section{NETWORK SETUP AND SIMULATION STUDY}

The network setup is used to analyze the performance of Light WiMAX considering Base Stations with Strict Priority Algorithms for channel allocation. Simulation is carried with one BS, and many SSs connected via wireless link. Downlink data transfer and various cases are taken to analyze the performance of BS and SSs. BS to SSs connections are created in downlink packet transmission. Two different bandwidth allocation techniques are used to allocate the channel bandwidth to multiple SSs which is gradually increases along with time for data transfer from BS to SSs. Picture given below.

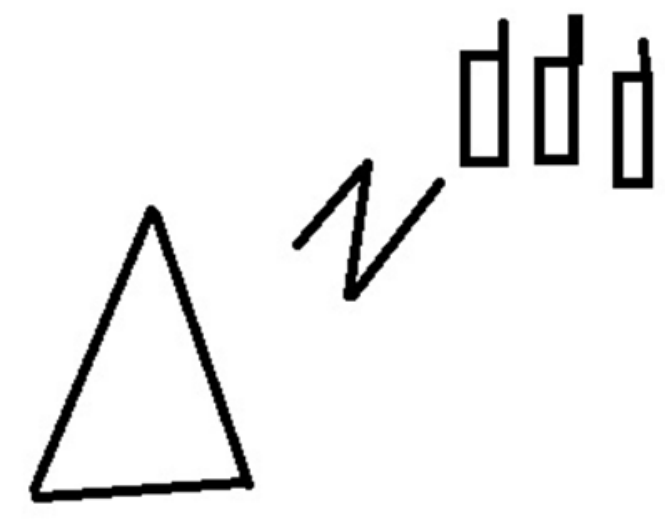

Fig. 1 Connection of Base Station with Subscriber Stations with SP

\section{SIMULATION PARAMETERS}

The performance analysis of Light WiMAX is done considering following parameters in table I:

\footnotetext{
Revised Manuscript Received on 16 September, 2019.

Khan Mubeen Ahmed, Mewar University, Department of Computer Science and Engineering, Gangarar, Rajasthan, India.

(Email: makkhan0786@gmail.com)

Bandhu Kailash Chandra, Mewar University, Department of Computer Science and Engineering, Gangarar, Rajasthan, India.

(Email: kailash_bandhu@yahoo.co.in)
} 
TABLE I. PARAMETERS USED FOR SIMULATION

\begin{tabular}{|c|c|}
\hline Parameters & Values \\
\hline Routing Protocols & AODV \\
\hline Transmission Protocol & TCP \\
\hline $\begin{array}{c}\text { Bandwidth Allocation } \\
\text { Algorithm }\end{array}$ & Strict Priority \\
\hline Simulation Time & $300 \mathrm{Sec}$ \\
\hline Number of Nodes & $10,20, \ldots \ldots . .100$ \\
\hline Frame Period & $0.005 \mathrm{M}-\mathrm{Sec}$ and \\
& $0.010 \mathrm{M}-\mathrm{Sec}$ \\
\hline
\end{tabular}

\section{PERFORMANCE METRICS}

The performance metrics are evaluated on the basis of three parameters given below:

- Throughput that measures the raw bytes sent by a source.

- Goodput that measures successfully received bytes.

- $\quad$ Packet Drop Rate

\section{RESULTS}

Throughput with No. of Nodes and Frame periods

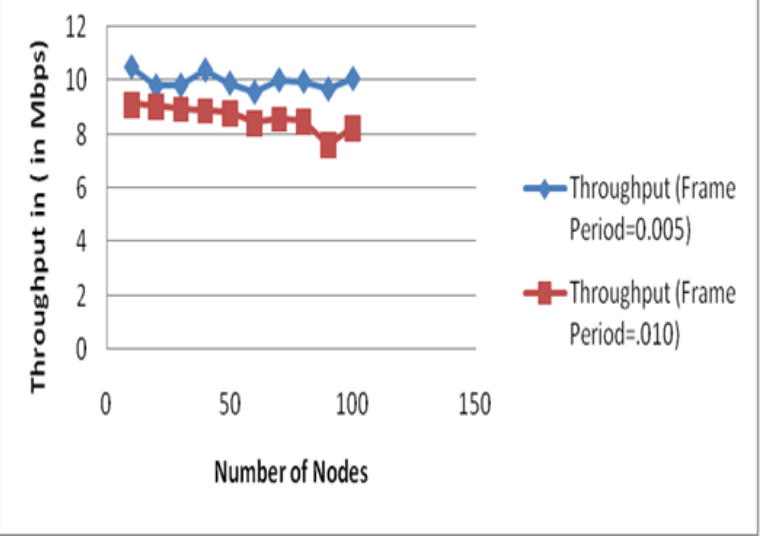

Fig. 2 Coverage of Relay station with distance and Throughput

It is observed from the graph shown in fig. 2 below that the value of throughput is obtained higher(10.516 and 9.12 Mbps) for frame period 0.005 and for 10 nodes. As number of nodes increases value of throughput decreases and obtained (10.09 and 8.24Mbps) for frame period 0.010 for 100 nodes. When frame period is less (i.e. 0.005 ) value of PDR increases with no of nodes because more and more frames could be transferred from the channel in the assigned time interval, and hence losses increases. When frame period is increased, lesser number of frames is transmitted and channel is busy all the time. Hence losses become lesser. A higher order modulation technique is useful for higher throughput with OFDM. As the number of subscriber station increases multiple bits are carried in a single OFDM symbol. All this is obtained since lesser frame period uses maximum bandwidth of channel and throughput is obtained higher [14].

When the data symbol is longer, the delay spread is a small and insignificant fraction of the symbol length, so the effect due to delay spread is minimized. When number of subscriber stations increases, full utilization of Base station happens and use wider channels. If this condition is satisfied, the system capacity may be increased

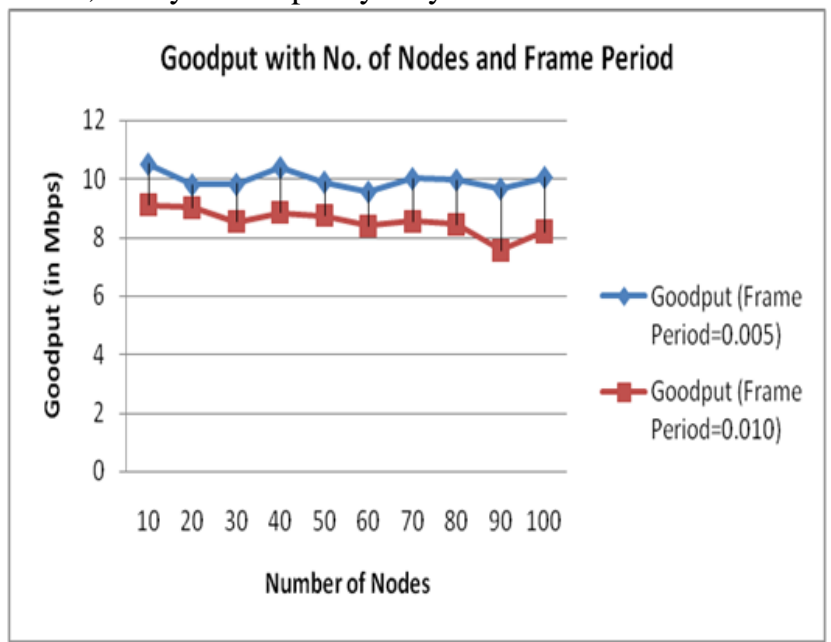

Fig. 3 Coverage of Base Station with Number of Nodes and Goodput

It is observed from the graph shown in fig 4 that the value of Goodput is also obtained higher when frame period is lesser (0.005) with lesser number of nodes for the connection. It is observed that the value of Goodput in obtained is $10.51 \mathrm{Mbps}$ and for 10 subscriber stations. When the same analysis is done with higher frame period (with 0.010) Goodput obtained is 9.11 Mbps. When Number of nodes increases the value of Goodput in both the cases decreases since dropping of packets occurs due to busy bandwidth of channel. In case of 100 Subscriber station it is obtained 10.06 Mbps with frame size $=0.005$ and 8.192 Mbps in case of frame size $=0.010$. This is due to the fact that as number of packets per second also increases, data transmission capacity of channel also increases and hence is obtained highest in lesser subscriber stations [13]. As number of nodes from base increases, it is observed that the coverage of base station ends and the Goodput becomes lesser due to business of the channel due to which drop rate increases. 
Packet Drop Rate with no. of nodes and Frame Period

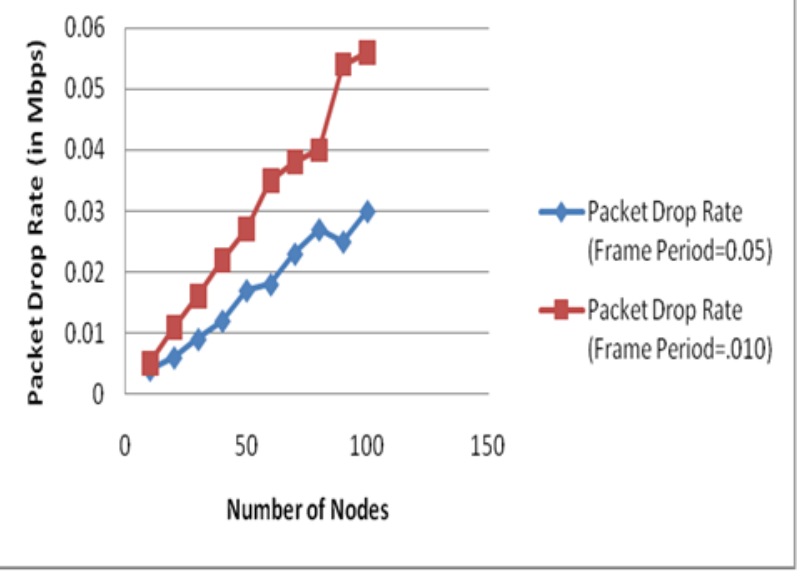

Fig. 4 Dropped Packets with Number of Nodes

It is observed that number of dropped packets is more as number of subscriber stations increases, due to the fact that when packets are sending then there is less possibility of packets to be lost since the signal is transmitting with maximum power from base station. As the traffic load increases with number of subscriber stations then calls are not serviced properly and are dropped after long waiting time [8]. It is observed that from fig. 4 below that Dropped packets are also increased since high modulation cannot be maintained over the entire length of the link or in a Non Line of sight environment. For such cases the error rates rises and the adaptive modulation feature drops the modulation to lower density modulation. Initially packet drop rate for 10 subscriber station is obtained 0.004 and $0.005 \mathrm{Mbps}$ in case of frame period 0.005 and 0.010 . As the number of nodes increases packet drop also increases (.ie 0.03 and0.05 Mbps) in case of frame period 0.005 and 0.010 . The data rates changes through the entire coverage area and depends on whether the reception is LOS or NLOS [12]. In case of NLOS reception the data rates drop significantly because of change of modulation [9].

\section{CONCLUSION}

In this paper the performance of WiMAX networks is analyzed with two 0.005 and 0.010 ) frame periods. Strict Priority is used in this paper for bandwidth allocation technique. The simulation results show that Increasing frame period decreases the performance of WiMAX Networks. So for efficient data transmission in WiMAX networks, lesser frame period will give better results. It is also analyzed that when number of nodes are lesser than higher throughput is obtained with lesser frame period and as the number of nodes increases value of throughput also decreases with higher frame period[14][15].

\section{FUTURE WORK}

Further studies can be carried out about the performance of base station with various other bandwidth allocation techniques with different types of parameters like frame delay, UL/DL frame symbols and with various other Services parameters. This whole analysis could also be done with other protocols and with IPV-6 version too.

\section{ACKNOWLEDGEMENT}

I am also thankful to my parents, my wife and the friends who coordinated me all the time. I am also thankful to SAGE University for downloading various articles to complete this research work. Lastly I am very much thankful to Mewar University, Gangrar Chittorgarh Rajasthan, India, along with the Research Guide and Hod of Mewar University without whom this research was not possible.

\section{REFERENCES}

1. ns2, http://www.isi.edu/nsnam/ns/

2. The WiMAX Handbook by Taylor and Francis Groupp127.www.taylor and francis.com IEEE 802.16 Working Group, "DRAFT Standard for Local and Metropolitan Area Networks Para 16: Air Interface for Broadband Wireless access Systems"IEEE P802.16Rev2/D1.Oct.2007

3. IEEE 802.16-2004, IEEE standard for local and Metropolitan area networks Part 16: Air interface for Broadband wireless Access Systems,http://standards.ieee.org/getieee.802/download/8 02-2004.pdf,October 1, 2004

4. Weighted round robin CPU Scheduling algorithm.pdf

5. William Stallings, PhD. "Operating Systems / Internal and Design Principals" ,pages 75-76, 406-408,Prantice Hall, 2001

6. Silbershatz, Galvin, and Gagane, "Operating System Concepts", pages 157-166, John Wiley and Sons, Inc.,2002

7. Harry Katzan, Jr., "Operating Systems / A Pragmatic Approach", pages 113,157,350-353, Van Nostrand Reinhold Company, 1973.

8. "Multiuser Communications, in IEEE conference on communications", vol. 1 p.331, IEEE Washington, DC 1995

9. A. Sayenko, O. Alanen, and T. Hamalainen, "Scheduling solution for the IEEE 802.16 base station," Int. J. Comp. and Telecommun. Netw., vol. 52, pp. 96 -115, Jan. 2008.

10. Pandey, M., Litoriya, R., Pandey, P. (2019) Novel Approach for Mobile Based App Development Incorporating MAAF. Wireless Personal Communications. DOI: 10.1007/s11277-019-06351-9

11. Pandey, M., Litoriya, R., Pandey, P.: Perception-Based Classification of Mobile Apps: A Critical Review. In: Smart Computational Strategies: Theoretical and Practical Aspects. Springer, Singapore (2019)121133.https://doi.org/10.1007/978-981-13-6295-8_11

12. https://www.google.com/search?ei=y0y3XLvBINn8rQG 2lq6QAg\&q =what + is + frame + period $+\% 3 \mathrm{~F} \&$ oq $=$ what + is + frame+period $+\& g s \_$l $=$psyab.1.1.35i3912j0i22i3013.234064.2384444..241832...0.0..0. 342.4625.0j8j12j1.....0....1...gwswiz.......0i71j0i131i67j0j0i131j0i67j0i20i263j0i10.UWoe ttBWBL8

13. Pandey M, Litoriya R and Pandey P (2018) An ISM approach for modeling the issues and factors of mobile app development. International Journal of Software Engineering and Knowledge Engineering 28 (7): $937-$ 953. 